\title{
The safety and efficacy of neoadjuvant PD-1 inhibitor with chemotherapy for locally advanced esophageal squamous cell carcinoma
}

\author{
Dijian Shen ${ }^{1,2}$, Qixun Chen ${ }^{1,2}$, Jie Wu ${ }^{1,2}$, Jianqiang Li $^{1,2}$, Kaiyi Tao ${ }^{1,2}$, Youhua Jiang ${ }^{1,2}$ \\ ${ }^{1}$ Department of Thoracic surgery, Cancer Hospital of the University of the Chinese Academy of Sciences (Zhejiang Cancer Hospital), Hangzhou, \\ China; ${ }^{2}$ Institute of Cancer and Basic Medicine (IBMC), the Chinese Academy of Science, Hangzhou, China \\ Contributions: (I) Conception and design: D Shen, Y Jiang, Q Chen; (II) Administrative support: Y Jiang, Q Chen; (III) Provision of study materials \\ or patients: J Wu, J Li, K Tao; (IV) Collection and assembly of data: D Shen, J Wu; (V) Data analysis and interpretation: D Shen, J Li, K Tao; (VI) \\ Manuscript writing: All authors; (VII) Final approval of manuscript: All authors. \\ Correspondence to: Youhua Jiang. Department of Thoracic surgery, Cancer Hospital of the University of the Chinese Academy of Sciences (Zhejiang \\ Cancer Hospital), No. 1 Banshan East Road, Gongshu District, Hangzhou 310022, China. Email: jiangyh@zjcc.org.cn.
}

\begin{abstract}
Background: Neoadjuvant therapy followed by esophagectomy has been recognized as an effective treatment for locally advanced esophageal cancer, though still has a dismal prognosis. Antibodies against programmed death 1 (PD-1) protein improve survival in patients with advanced or metastatic esophageal squamous cell carcinoma (ESCC) compared with chemotherapy in second-line therapy. However, neoadjuvant PD-1 inhibitor combined with chemotherapy has not been tested in locally advanced ESCC. We conducted this study to evaluate the efficacy and safety of pd-1 inhibitor in neoadjuvant chemotherapy.

Methods: In this study, we administered 28 adults with untreated, surgically resectable locally advanced ESCC. PD-1 inhibitor with chemotherapy [albumin paclitaxel $100 \mathrm{mg} / \mathrm{m}^{2}$ on days 1 and $8+$ carboplatin with an area under the curve (AUC) of 5 on day 1] were administered every 3 weeks intravenously, and surgery was performed approximately 3-5 weeks after the second dose. The primary purpose of the study was to evaluate the feasibility and safety of this regimen.
\end{abstract}

Results: In all, 28 locally advanced ESCC patients were enrolled, 27 patients received surgery, 9 (33.3\%) patients' postoperative pathological specimens suggested pCR, and 11 (40.7\%) patients' primary tumor suggested complete response. Neoadjuvant PD-1 inhibitor with chemotherapy had an acceptable side-effect profile, 26 patients' tumors were completely resected (96.3\% were R0). According to the RESIST v.1.1, the response in all 27 patients was evaluated by a computed tomography (CT) scan before surgery, showing 12 patients with complete response (CR), 12 with partial response (PR), and 3 with stable disease (SD). For surgical procedures, 15 (55.6\%) patients underwent minimal invasive surgery, 4 (14.8\%) underwent right transthoracic open esophagectomy, and 8 (29.6\%) underwent hybrid approaches.

Conclusions: The novel treatment of PD-1 inhibitor with chemotherapy in the neoadjuvant setting for locally advanced ESCC produced satisfactory outcomes: an unprecedentedly high pCR rate for neoadjuvant chemotherapy, a high R0 resection rate, and a low-toxicity profile were achieved. The long-term efficiency of this novel treatment and the validity of the present findings should be confirmed with longer follow-up and prospective comparative trials.

Keywords: Neoadjuvant immunotherapy; esophageal squamous cell carcinoma (ESCC); thoracic surgery; programmed death 1 (PD-1)

Submitted Oct 23, 2020. Accepted for publication Feb 08, 2021.

doi: 10.21037/jgo-20-599

View this article at: http://dx.doi.org/10.21037/jgo-20-599

$\wedge$ ORCID: 0000-0001-7132-7885. 


\section{Introduction}

Esophageal cancer is the seventh most common malignancy, and the sixth most common cause of cancer death in the world (1). In 2018, a total of 572,000 new cases of were diagnosed, with 509,000 deaths being caused by this disease (1). Esophageal cancer can be broadly divided into two histologic subtypes: esophageal adenocarcinoma and esophageal squamous cell carcinoma (ESCC). The most frequent histologic subtype worldwide is ESCC, accounting for $87 \%$ of all esophageal cancers (2). Esophagectomy is still the main treatment for esophageal cancer. However, in patients with locally advanced esophageal cancer, surgery alone is often associated with a high recurrence and metastasis rate. In order to improve survival and reduce local and distant recurrence, neoadjuvant therapy has come into use. Neoadjuvant therapy is the administration of therapeutic agents before a main treatment. The purpose is to reduce the size or scope of the tumor before the use of radical treatment intervention, so as to make the operation easier and more likely to be successful, and reduce the consequences of a wider range of treatment techniques. If the size or scope of the tumor is not reduced, a wider range of treatment techniques are needed $(3,4)$. Based on the results of the CROSS trail, neoadjuvant chemoradiotherapy (nCRT) plus surgery has been selected as a standard treatment for patients with locally advanced esophageal cancer in most parts of the Western world (5). In, Asia, however, according to the JCOG9907 trial, neoadjuvant chemotherapy (nCT) is advocated as a standard treatment, especially in Japan (6).

Antibodies against the immune inhibitory pathway of programmed death 1 (PD-1) protein represent a relatively modern innovation in the treatment of malignant tumors (7), showing particular efficacy in patients with advanced or metastatic ESCC.

Compared with chemotherapy, second-line PD-1 inhibitors have been shown to significantly improve overall survival (OS) in patients, while boasting a manageable safety profile (8-10). Owing to these and similar studies, PD-1 inhibitors have emerged as the new optimal secondline treatment choice for advanced or metastatic esophageal cancer patients.

One preoperative study found that after two doses of PD-1 blockers were used in patients with early stage lung cancer, there were few immediate adverse reactions, the planned operation was not delayed, and $45 \%$ of tumors demonstrated major evaluable pathological reactions (MPR) (11).
Meanwhile, the NADIM (neoadjuvant chemotherapy and nivolumab in resectable non-small-cell lung cancer) study, another clinical phase II trail (NCT03838159), evaluated patients treated with chemotherapy for 3 cycles every 21 days as neoadjuvant treatment followed by surgery; the patients showed higher MPR and pathological complete response (pCR) rates after receiving $\mathrm{PD}-1$ inhibitor, In addition, Radical esophagectomy for ESCC after neoadjuvant chemoradiation therapy with pembrolizumab may not increase the operative risk or reduce the quality of radical dissection including lymphadenectomy (12).

PD-L1 expression is enriched in ESCC, which might increase the sensitivity of these patients to tumor elimination after immunosuppression (13). No neoadjuvant approaches using immune inhibitors have thus far been reported for the treatment of locally advanced ESCC. We therefore performed this prospective pilot study to evaluate the treatment response and safety of the use of neoadjuvant PD-1 inhibitor with chemotherapy in a group of patients with locally advanced ESCC. We present the following article in accordance with the TREND reporting checklist (available at http://dx.doi.org/10.21037/jgo-20-599).

\section{Methods}

\section{Study design}

This single-group study was designed by the authors and conducted at the Cancer Hospital of the University of the Chinese Academy of Sciences (Zhejiang Cancer Hospital). The patients received 2 doses of intravenous PD-1 inhibitor (nivolumab at a dose of $3 \mathrm{mg}$, pembrolizumab at a dose of $2 \mathrm{mg}$ per kilogram of body weight, and camrelizumab at a dose of $200 \mathrm{mg}$ ) every 3 weeks, with 2 cycles of chemotherapy being simultaneously performed. Premedication with dexamethasone was not expected in this patient group, so the detailed regimen proceeded as follows: albumin paclitaxel $100 \mathrm{mg}$ per square meter of body-surface area on days 1 and 8, and carboplatin targeted at an area under the curve (AUC) of $5 \mathrm{mg}$ per milliliter per minute on day 1 . The surgery was performed approximately 3-5 weeks after the second dose. Changes in tumor size were evaluated according to response evaluation criteria in solid tumors (RECIST), version 1.1 (14). The primary end points were safety and feasibility. The patients were closely monitored for toxic effects of neoadjuvant therapy with the use of the NCICTC for Adverse Events version 5.0. The key secondary and exploratory end points were pathological 
responses to treatment. To grade the response to therapy, we classified the degree of degeneration of histomorphology into the following four categories: grade 0 , no residual cancer cells (pCR); grade 1, single cells or small groups of cancer cells; grade 2, residual cancer cells outgrown by fibrosis; and grade 3 , minimum or no treatment effect $(15,16)$.

\section{Patients}

The key inclusion criteria were the following: patients with histologically confirmed, potentially curable ESCC with cT1N1-3M0 or cT2-4aN0-3M0 (Union for International Cancer Control TNM Classification $8^{\text {th }}$ Edition); tumors located in the thoracic esophagus; no metastatic cervical lymph nodes or prior therapy for any cancer; 20-80 years of age; Eastern Cooperative Oncology Group (ECOG) performance status of 0 or 1 ; normal organ function; adequate pulmonary and cardiac function (17); and written consent provided. The key exclusion criteria were immunodeficiency disease, ongoing systemic immunosuppressive therapy with either corticosteroids (>10 $\mathrm{mg}$ daily prednisolone equivalent) or other immunosuppressive drugs, infectious disease, clinically significant concurrent cancer, inability to undergo gastric tube reconstruction after esophagectomy, and hypersensitivity to albumin paclitaxel and carboplatin drugs.

\section{Surgery}

After neoadjuvant therapy, patients received the same examinations as those given pretreatment. After neoadjuvant therapy, endoscopy evaluation is not necessary. Minimally invasive esophagectomy (MIE), right transthoracic open esophagectomy, or hybrid approaches (use of videoassisted thoracoscopy and laparotomy) with a total 2-field lymphadenectomy were performed. A gastric tube was used to reconstruct the digestive tract after esophagectomy. Transesophageal or left thoracic esophagectomy was not performed due the limited lymph node dissection capacity afforded by of these two methods, especially for the lymph nodes along the bilateral recurrent laryngeal nerve. All operations were performed by five attending surgeons in our center, each with more than 100 cases of esophageal cancer surgery experience. Postoperative complications including hematological toxicity, anastomotic leak, pleural effusion, and lung infection were recorded on the case report form for up to 90 days after surgery, Patients were followed up every 3 months in the first year after the end of treatment, and every 6 months in the second year.

\section{Study oversight}

This study was approved by the institutional review boards at the Cancer Hospital of The University of the Chinese Academy of Sciences (Zhejiang Cancer Hospital): IRB2020-192. Written informed consent was obtained from the patients. This study is conformed to the provisions of the Declaration of Helsinki (as revised in 2013). The study was designed and written by the authors, who ensure the accuracy and completeness of the reported data and compliance with study protocol. Only the authors participated in the writing of the manuscript, and other companies or institutions had no role in the research and reporting.

\section{Statistical analysis}

Adverse events, Side effects and feasibility were continuously monitored. We assumed that treatment was not feasible if more than $25 \%$ of patients had a $90 \%$ or higher probability of delayed surgery. We also determined that treatment was not safe if the risk of grade 3 or 4 toxicity exceeded $25 \%$. The mean follow-up time was calculated by using the reverse Kaplan-Meier method. The range of follow-up included all follow-up time. We also used the Kaplan-Meier method to calculate recurrence-free survival (RFS) from the date of surgery to recurrence or death. The $P$ value was bilateral, and the significance level was set at 0.05 for all analyses unless otherwise indicated.

\section{Results}

\section{Patient characteristics}

From June 1, 2019 through June 30, 2020, we enrolled 28 patients, all of whom completed the two cycles of neoadjuvant therapy. One patient refused operation after neoadjuvant treatment and received radical radiotherapy instead. The baseline characteristics of these patients were eligible for inclusion in the study (Table 1). The median age was 62.2 years, 4 patients (14.3\%) were stage II, $21(75.0 \%)$ were stage III, and $3(10.7 \%)$ were stage IVA disease. Tumors were located in the proximal third of the esophagus in 3 $(10.7 \%)$ patients, the middle third in $14(50 \%)$ patients, the distal third in $8(28.6 \%)$ patients, and the esophagogastric 
Table 1 Patient characteristics at baseline

\begin{tabular}{|c|c|}
\hline Characteristic & Value \\
\hline \multicolumn{2}{|l|}{ Age, yr } \\
\hline Median & 62.2 \\
\hline Range & $48-79$ \\
\hline Male sex, n (\%) & $27(96.4)$ \\
\hline \multicolumn{2}{|l|}{ Tumor location, n (\%) } \\
\hline Proximal third & $3(10.7)$ \\
\hline Middle third & $14(50.0)$ \\
\hline Distal third & $8(28.6)$ \\
\hline Esophagogastric junction & $3(10.7)$ \\
\hline \multicolumn{2}{|l|}{ Clinical T stage, n (\%) } \\
\hline cT1 & 0 \\
\hline cT2 & $3(10.7)$ \\
\hline сT3 & $23(82.1)$ \\
\hline cT4a & $2(7.1)$ \\
\hline \multicolumn{2}{|l|}{ Clinical N stage, n (\%) } \\
\hline NO & $2(7.1)$ \\
\hline N1 & $15(53.6)$ \\
\hline N2 & $9(32.1)$ \\
\hline N3 & $2(7.1)$ \\
\hline \multicolumn{2}{|l|}{ Performance status (ECOG) } \\
\hline 0 & 25 (89.3) \\
\hline 1 & $3(10.7)$ \\
\hline
\end{tabular}

junction in $3(10.7 \%)$ patients; $71.4 \%$ were current or former smokers, and $78.6 \%$ had a drinking history.

\section{Safety and feasibility of neoadjuvant therapy}

All patients completed two cycles of PD-1 inhibitor with chemotherapy, with none being withdrawn from the course of neoadjuvant therapy due to toxic effects or disease progression. This combined neoadjuvant treatment was not associated with any previously unreported toxic effects. Of the 28 patients, $16(57.1 \%)$ experienced treatmentrelated adverse events (Table 2), 2 (7.1\%) experienced grade 3 events with 1 patient rush (Figure 1), and 1 patient thrombocytopenia. No grade $\geq 4$ adverse events were observed. The two grade 3 adverse event patients quickly
Table 2 Adverse events during neoadjuvant chemoradiotherapy

\begin{tabular}{lcc}
\hline $\begin{array}{l}\text { Events during neoadjuvant therapy, } \\
\text { No. of patients (\%) }\end{array}$ & Grade 1-2 & Grade 3 \\
\hline White blood cell count decreased & $5(17.8)$ & - \\
Neutrophil count decreased & $3(10.7)$ & - \\
Anemia & $3(10.7)$ & - \\
Thrombocytopenia & $2(7.1)$ & $1(3.6)$ \\
Nausea & $4(14.3)$ & - \\
Decreased appetite & $4(14.3)$ & - \\
Diarrhea & $2(7.1)$ & - \\
Constipation & $3(10.7)$ & - \\
Fatigue & $3(10.7)$ & - \\
Alopecia & $7(25.0)$ & - \\
Rash & $2(7.1)$ & $1(3.6)$ \\
Reactive cutaneous capillary & $4(14.3)$ & - \\
endothelial proliferation & & - \\
Neurotoxic effects & $2(7.1)$ & - \\
Hypothyroidism & $1(3.6)$ & - \\
\hline
\end{tabular}

recovered after hormone therapy and platelet transfusion. Computed tomography (CT) was performed in 27 patients before surgery to evaluate the treatment effect: $12(44.4 \%)$ patients showed complete response (CR), 12 (44.4\%) patients showed partial response (PR), 3 (11.1\%) patients showed stable disease, and none showed disease progression.

\section{Surgery}

The election of surgery was decided upon by patients, with a total of 27 patients ultimately undergoing surgery. The 1 patient who refused surgery eventually underwent radical concurrent chemoradiotherapy. In the surgery $26(96.3 \%)$ patients underwent complete resection (R0), while 1 patient failed to undergo radical resection due to retroperitoneal lymph node metastasis. The proportions of the three surgical approaches are as follows: 15 (55.6\%) patients underwent MIE esophagectomy, with 1 procedure being robotically assisted, $4(14.8 \%)$ being open right thoracotomy, and 8 (29.6\%) being hybrid approaches. The mean operative time was 194 minutes (ranging from 110 to $320 \mathrm{~min}$ ) which is similar to the operation time for esophageal cancer without neoadjuvant treatment.

The postoperative complications are summarized in 


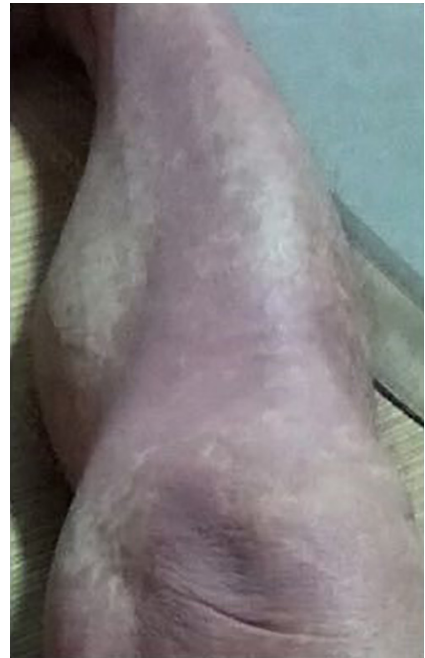

pretreatment imaging

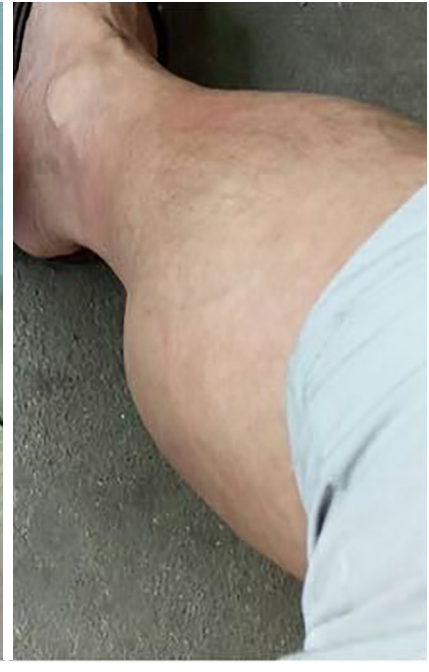

1 week dexamethasone
Figure 1 A comparative picture of one patient's skin with degree 3 rash before and after treatment.

Table 3 Postoperative complications

\begin{tabular}{lc}
\hline Postoperative events & No. of patients (\%) \\
\hline Anastomotic leakage & $5(18.5)$ \\
Chylothorax & $2(7.4)$ \\
Pulmonary complications & $3(11.1)$ \\
Pleural effusion & $4(14.8)$ \\
Recurrent nerve paralysis & $2(7.4)$ \\
Atrial fibrillation & $2(7.4)$ \\
Ascites & $1(3.7)$ \\
Hematology-related complications & $2(7.4)$ \\
\hline
\end{tabular}

Table 3. No patients experienced intraoperative complications or died in hospital. During the postoperative periods, the most common complications were anastomotic leakage $(\mathrm{n}=5,18.5 \%)$, pleural effusion $(\mathrm{n}=4,14.8 \%)$, and pneumonia $(n=3,11.1 \%)$. No significant differences in the occurrence of complications were found when compared with previous studies (5). One patient was required to undergo reoperation because of uncontrolled chylothorax. The mean followup was 6 months (range, 1-12 months). Of the 26 patients who underwent R0 resection, 25 (96.2\%) survived without recurrence. Only 1 case had anastomotic recurrence 5 months after operation.

\section{Pathological assessment}

Among the 27 patients who underwent surgery, a median of 24 (range from 9 to 46) lymph nodes were resected in patients. pCR (ypT0N0; y denotes underwent neoadjuvant therapy, and $\mathrm{p}$ denotes pathological assessment) was seen in the resection specimens of $9(33.3 \%)$ patients, and in the primary tumor of $11(40.7 \%)$ patients.

The pathological tumor regression in all resection specimens compared with CT evaluation is listed in Figure 2. In contrast with the evaluation of the neoadjuvant PD-1 treatment in lung cancer (11), our preoperative CT evaluation is basically consistent with the final pathological evaluation (Figure 3).

\section{Discussion}

Compared with surgery or adjuvant therapy alone, neoadjuvant therapy (mainly chemotherapy or chemoradiotherapy) can improve the survival rate of patients with esophageal or esophagogastric junction cancer $(5,6)$. Thus, neoadjuvant therapy plus esophagectomy has been adopted as a standard treatment strategy for patients with potentially curable locally advanced esophageal cancer. However, with the good effect of immune checkpoint inhibitors (ICIs) being observed in many kinds of cancer, including melanoma (18), head and neck squamous cell cancer (19), non-small cell lung cancer (20), renal cell cancer (21), urothelial cancer (22), and relapsed or refractory Hodgkin's lymphoma (23), further experiments with a neoadjuvant therapy regimen of lower toxicity and better effect in the treatment of esophageal cancer are needed. Neoadjuvant PD-1 inhibitor therapy for lung cancer has already been evaluated; for instance, one study found that only two administrations of nivolumab monotherapy could achieve MPR in $45 \%$ of patients with resectable non-small cell lung cancer (11). However, it is still unclear whether neoadjuvant ICIs should be combined with chemotherapy or chemoradiotherapy, or which regimen will have a better effect.

Radiation induces chemokines to attract effector $\mathrm{T}$ cells to tumor and vascular adhesion molecules, promoting $\mathrm{T}$ cell infiltration. This process is called immunogenic regulation, which not only plays a role in tumor regression after radiotherapy, but also functions to promote adaptive antitumor immunity (24) with immunogenic modulation 


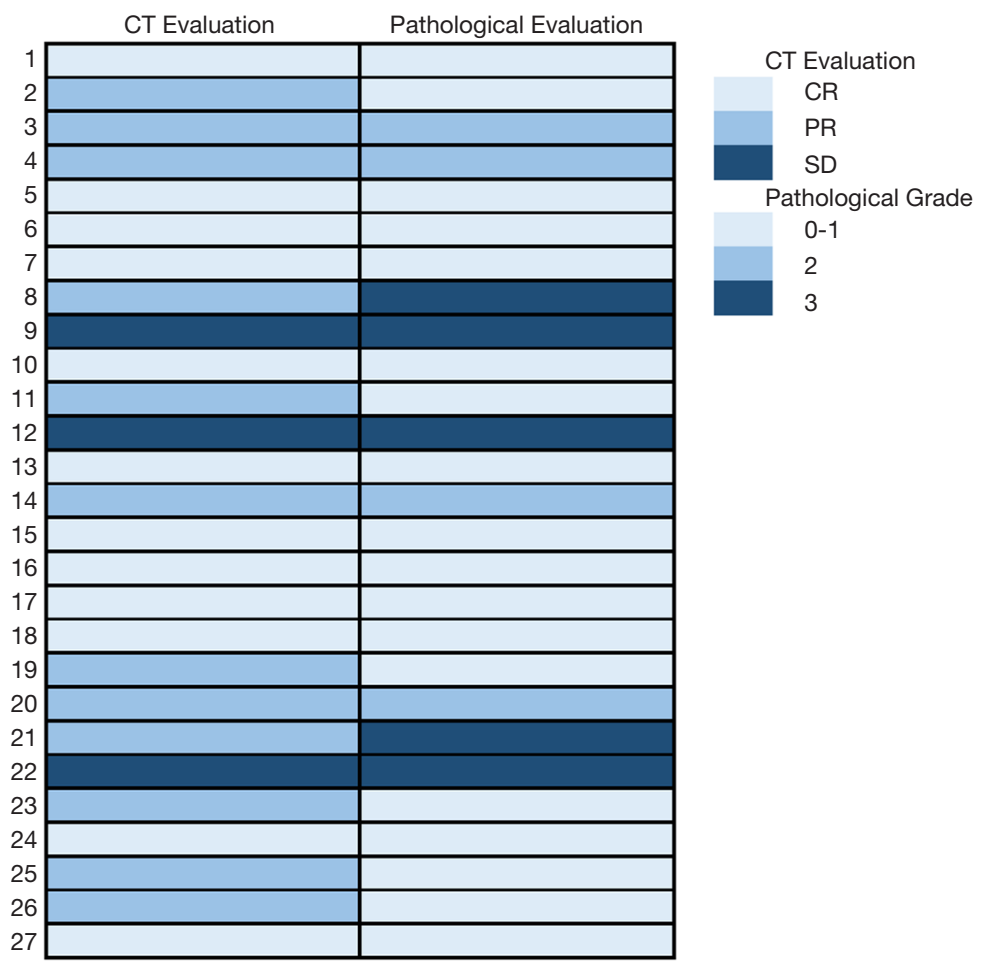

Figure 2 Preoperative CT evaluation compared with the final pathological evaluation. The degree of degeneration of histomorphology: grade 0 , no residual cancer cells (pCR); grade 1, single cells or small groups of cancer cells; grade 2, residual cancer cells outgrown by fibrosis; and grade 3, minimum or no treatment effect. CT evaluation: CR, complete response; PR, partial response; SD, stable disease.

being able to enhance the effect of ICIs. We have seen the effect of this combination therapy in lung cancer (25). In one study, 709 locally advanced, unresectable, non-small cell lung cancer patients received consolidation therapy (473 received durvalumab and 236 received placebo) after definitive chemoradiotherapy. The median progression-free survival (PFS) of the durvalumab-treated patients was 16.8 versus 5.6 months with those treated with placebo. The latest data also show higher 3-year survival rates for the durvalumab group. However, the concurrent use radiotherapy with ICI, remains controversial. Recently, low-dose radiation was used to treat novel coronavirus 2019 (COVID-19) pneumonia through suppressing the immune cytokine storm (26) in oxygen-dependent patients, which rapidly improved their clinical status. However, concurrent radiotherapy may be a double-edged sword, it can kill tumor cells, as it may also cause the decrease of T cells activated by PD-1 blockade due to the depletion of immune cytokines such as interleukin-2 (IL-2) and interferon gamma (IFN- $\gamma$ ) (27).

In this single-center prospective clinical trial, we administered a neoadjuvant treatment regimen of PD1 blockade combined with chemotherapy for patients with potentially curable locally advanced ESCC. We observed that the use of two cycles of neoadjuvant therapy in patients with esophageal cancer was associated with fewer direct and severe adverse events, did not delay planned surgery, and resulted in $33.3 \%$ of tumors being eligible for pCR. No serious immune-related adverse events, such as pneumonia, myocarditis, or hepatitis, were observed in our patients, which may be related to the good physical condition of our patients compared with advanced or metastatic patients. The rate of pCR was much better than that of neoadjuvant chemotherapy and comparable to that of nCRT (5). For our surgeons, another advantage of this regimen is that it does not increase the difficulty of surgery. Our mean operative time was 194 minutes, which is comparable to the operation time for esophageal cancer without neoadjuvant treatment.

Furthermore, we observed that after this neoadjuvant treatment, most of the esophageal tumors tended to more loosely adhere to the surrounding tissues permitting easier removal, which is different from the condition observed in patients after radiotherapy or after neoadjuvant therapy 
A Grade 0 Patient 16

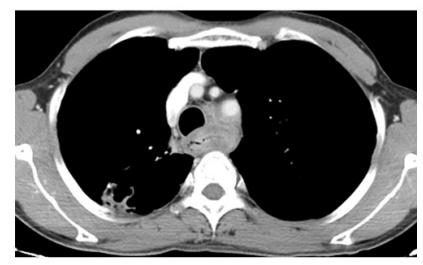

Pretreatment Imaging

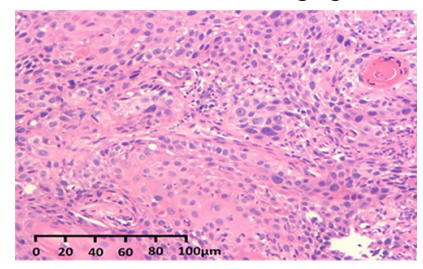

Pretreatment Tumor Biopsy

B Grade 1 Patient 18

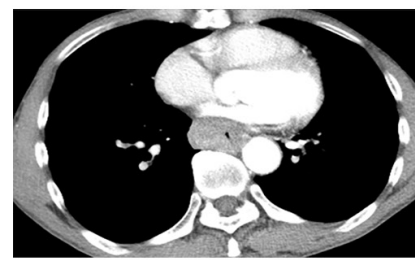

Pretreatment Imaging

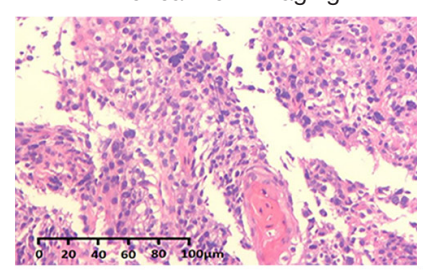

Pretreatment Tumor Biopsy

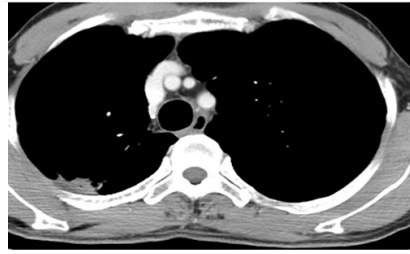

Preoperative Imaging

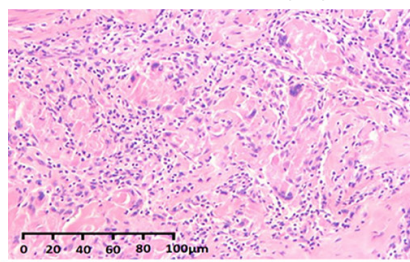

Resection Specimen

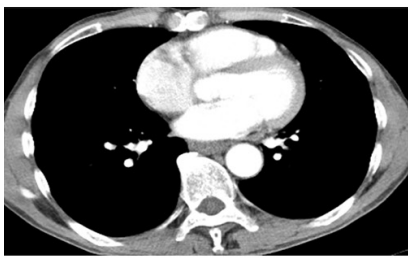

Preoperative Imaging

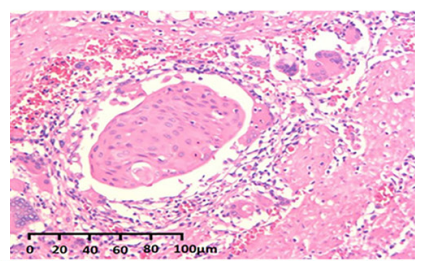

Resection Specimen

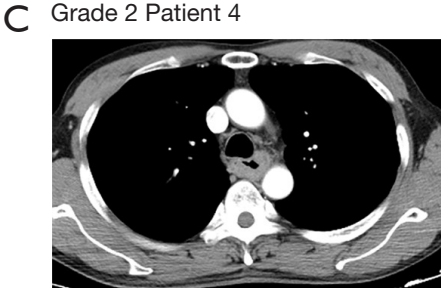

Pretreatment Imaging

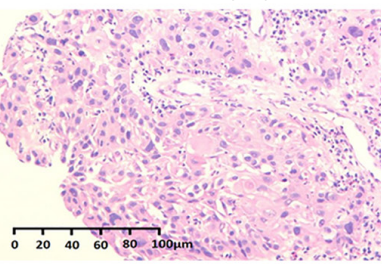

Pretreatment Tumor Biopsy

Grade 3 Patient 9

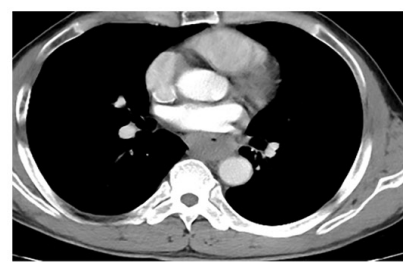

Pretreatment Imaging

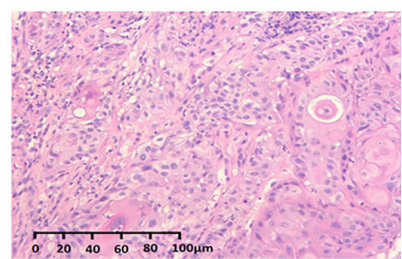

Pretreatment Tumor Biopsy

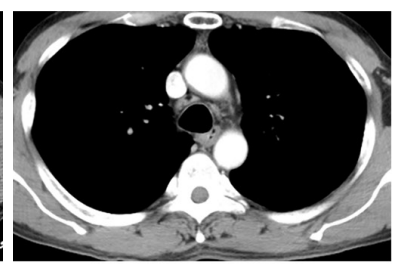

Preoperative Imaging

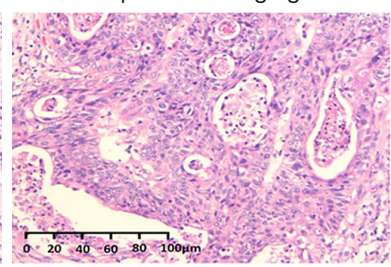

Resection Specimen

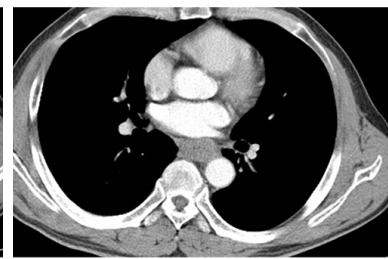

Preoperative Imaging

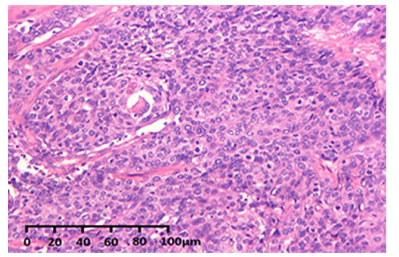

Resection Specimen

Figure 3 Patterns of Pathological and Radiologic Response to Neoadjuvant Therapy with pathological degeneration of grade 0-grade 3. (A) Patient 16 with grade 0 degeneration of histomorphology. In the upper row, the CT scan shows pretreatment and preoperative imaging. CT performed preoperative shows complete response with tumor. In the lower row, representative sections of tumor specimens which was obtained before treatment (left) and after the administration (right) (hematoxylin and eosin staining). (B) Patient 18 with grade 1 degeneration of histomorphology. CT performed preoperative also shows complete response with tumor. In the lower row, representative sections of tumor specimens which was obtained before treatment (left) and after the administration (right) (hematoxylin and eosin staining). (C) Patient 4 with grade 2 degeneration of histomorphology. In the upper row, the CT scan shows pretreatment and preoperative imaging. CT performed preoperative shows partial response with tumor. In the lower row, representative sections of tumor specimens which was obtained before treatment (left) and after the administration (right) (hematoxylin and eosin staining). (D) Patient 9 with grade 3 degeneration of histomorphology. CT performed preoperative shows stable disease with tumor. CT performed preoperative shows partial response with tumor. In the lower row, representative sections of tumor specimens which was obtained before treatment (left) and after the administration (right) (hematoxylin and eosin staining).

for lung cancer. The NEOSTAR trial (NCT03158129) indicated that some patients have increased difficulty in surgery because it is more challenging to separate blood vessels after hilar fibrosis, but fibrosis in the adventitia of the esophagus is rarely seen with these patients. This may suggest that the response to ICIs varies across different cancer types.

Another interesting finding in our trial is that no pseudoprogression was observed. It is understood that some patients will have pseudoprogression of the tumor after ICI 
treatment. In the Checkmate-159 trial, the volume of tumor increased preoperatively evaluated by CT in two patients, and there was no or the negligible amounts of residual tumor in surgical specimens (11). When we combined PD-1 and chemotherapy, our presurgical CT scans were basically consistent with those of the final pathological evaluation. In addition to the chemotherapy itself killing tumor cells, a further effect may be that the host immune system is activated by chemotherapy, which helps in the tumor degeneration process (28). Chemotherapy may also help to activate tumor-specific $\mathrm{T}$ cells by promoting tumor antigen presentation after cancer cell death and by destroying immunosuppressive factors $(29,30)$. Therefore, the combination of appropriate chemotherapy drugs and PD-1 blockers can improve the efficacy of PD-1 blockers, especially for tumors with weak immunogenicity and poor chemosensitivity (31).

Although our study produced relatively promising results, some issues are still a concern. One of these is the number of cycles of neoadjuvant therapy. For lung cancer, several large-sample phase 3 trials have extended the treatment cycle to 3-4 cycles (ClinicalTrials.gov No. NCT02998528, NCT03425643, NCT03456063, and NCT03800134). It is unclear, however, whether this increased number of cycle can improve the therapeutic effect and yield a better pCR rate, and whether it would increase the toxicity and side effects. Secondly, whether patients who achieve pCR still need postoperative adjuvant treatment or simply require regular observation, still needs to be determined, while the better choice of adjuvant regimen-PD-1 blockade maintenance treatment or combination with chemotherapy-is not known. Thirdly, we are presently unable to screen for those patients who will receive the maximum benefit from neoadjuvant immunotherapy with chemotherapy, and predictive biomarkers urgently need to be identified. Finally, we cannot confirm that a high postoperative pCR rate necessarily entails a high survival rate. Although our follow-up has revealed no cases of recurrence in pCR patients thus far, the follow-up time has been too short to offer conclusive results. The only relapsed patient had grade 3 pathological regression in our trial.

Indeed, the limitations of our study include, but are not limited to, the short postoperative follow-up time and the small number of patients enrolled. In order to determine the benefit and safety of neoadjuvant therapy, more prospective studies on the expected efficacy of neoadjuvant therapy are needed.

\section{Acknowledgments}

Funding: The study was supported by Medical Health Science and Technology Project of Zhejiang Provincial Health Commission (grant number: 2020366703).

\section{Footnote}

Reporting Checklist: The authors have completed the TREND reporting checklist. Available at http://dx.doi. org/10.21037/jgo-20-599

Data Sharing Statement: Available at http://dx.doi. org/10.21037/jgo-20-599

Conflicts of Interest: All authors have completed the ICMJE uniform disclosure form (available at http://dx.doi. org/10.21037/jgo-20-599). The authors have no conflicts of interest to declare.

Ethical Statement: The authors are accountable for all aspects of the work in ensuring that questions related to the accuracy or integrity of any part of the work are appropriately investigated and resolved. This study was approved by the institutional review boards at the Cancer Hospital of The University of the Chinese Academy of Sciences (Zhejiang Cancer Hospital): IRB-2020192. Written informed consent was obtained from the patients. This study is conformed to the provisions of the Declaration of Helsinki (as revised in 2013).

Open Access Statement: This is an Open Access article distributed in accordance with the Creative Commons Attribution-NonCommercial-NoDerivs 4.0 International License (CC BY-NC-ND 4.0), which permits the noncommercial replication and distribution of the article with the strict proviso that no changes or edits are made and the original work is properly cited (including links to both the formal publication through the relevant DOI and the license). See: https://creativecommons.org/licenses/by-nc-nd/4.0/.

\section{References}

1. Bray F, Ferlay J, Soerjomataram I, et al. Global cancer statistics 2018: GLOBOCAN estimates of incidence and mortality worldwide for 36 cancers in 185 countries. CA Cancer J Clin 2018;68:394-424.

2. Thrift AP. The epidemic of oesophageal carcinoma: where 
are we now? Cancer Epidemiol 2016;41:88-95.

3. Kitagawa Y, Uno T, Oyama T, et al. Esophageal cancer practice guidelines 2017 edited by the Japan esophageal society: part 1. Esophagus 2019;16:1-24.

4. Ajani JA, D'Amico TA, Bentrem DJ, et al. Esophageal and Esophagogastric junction cancers, version 2.2019, NCCN clinical practice guidelines in oncology. J Natl Compr Canc Netw 2019;17:855-83.

5. van Hagen P, Hulshof MC, van Lanschot JJ, et al. Preoperative chemoradiotherapy for esophageal or junctional cancer. N Engl J Med 2012;366:2074-84.

6. Ando N, Kato H, Igaki H, et al. A randomized trial comparing postoperative adjuvant chemotherapy with cisplatin and 5 -fluorouracil versus preoperative chemotherapy for localized advanced squamous cell carcinoma of the thoracic esophagus (JCOG9907). Ann Surg Oncol 2012;19:68-74.

7. Topalian SL, Hodi FS, Brahmer JR, et al. Safety, activity, and immune correlates of anti-PD-1 antibody in cancer. N Engl J Med 2012;366: 2443-54.

8. Huang J, Xu J, Chen Y, et al. Camrelizumab versus investigator's choice of chemotherapy as second-line therapy for advanced or metastatic oesophageal squamous cell carcinoma (ESCORT): a multicentre, randomised, open-label, phase 3 study. Lancet Oncol 2020;21:832-42.

9. Kato K, Cho BC, Takahashi M, et al. Nivolumab versus chemotherapy in patients with advanced oesophageal squamous cell carcinoma refractory or intolerant to previous chemotherapy (ATTRACTION-3): a multicentre, randomised, open-label, phase 3 trial. Lancet Oncol 2019;20:1506-17.

10. Shah MA, Kojima T, Hochhauser D, et al. Efficacy and Safety of Pembrolizumab for Heavily Pretreated Patients With Advanced, Metastatic Adenocarcinoma or Squamous Cell Carcinoma of the Esophagus: The Phase 2 KEYNOTE-180 Study. JAMA Oncol 2019;5:546-50.

11. Forde PM, Chaft JE, Smith KN, et al. Neoadjuvant PD-1 Blockade in Resectable Lung Cancer. N Engl J Med 2018;378:1976-86.

12. Park SY, Hong MH, Kim HR, et al. The feasibility and safety of radical esophagectomy in patients receiving neoadjuvant chemoradiotherapy with pembrolizumab for esophageal squamous cell carcinoma. J Thorac Dis 2020;12:6426-34.

13. Rong L, Liu Y, Hui Z, et al. PD-L1 expression and its clinicopathological correlation in advanced esophageal squamous cell carcinoma in a Chinese population. Diagn
Pathol 2019;14:6.

14. Eisenhauer EA, Therasse P, Bogaerts J, et al. New response evaluation criteria in solid tumours: revised RECIST guideline (version 1.1). Eur J Cancer 2009;45:228-47.

15. Mandard AM, Dalibard F, Mandard JC, et al. Pathologic assessment of tumor regression after preoperative chemoradiotherapy of esophageal carcinoma: clinicopathologic correlations. Cancer 1994;73:2680-6.

16. Chirieac LR, Swisher SG, Ajani JA, et al. Posttherapy pathologic stage predicts survival in patients with esophageal carcinoma receiving preoperative chemoradiation. Cancer 2005;103:1347-55.

17. Oken MM, Creech RH, Tormey DC, et al. Toxicity and response criteria of the Eastern Cooperative Oncology Group. Am J Clin Oncol 1982;5:649-55.

18. Hamid O, Robert C, Daud A, et al. Safety and tumor responses with lambrolizumab (anti-PD-1) in melanoma. N Engl J Med 2013;369:134-44.

19. Seiwert TY, Burtness B, Mehra R, et al. Safety and clinical activity of pembrolizumab for treatment of recurrent or metastatic squamous cell carcinoma of the head and neck (KEYNOTE-012): an open-label, multicentre, phase 1b trial. Lancet Oncol 2016;17:956-65.

20. Gadgeel SM, Stevenson J, Langer CJ, et al. Pembrolizumab (pembro) plus chemotherapy as front-line therapy for advanced NSCLC: KEYNOTE-021 cohorts A-C. J Clin Oncol 2016;34:abstr 9016.

21. Motzer RJ, Sharma P, McDermott DF, et al. CheckMate 025 phase III trial: outcomes by key baseline factors and prior therapy for nivolumab (NIVO) versus everolimus (EVE) in advanced renal cell carcinoma (RCC). J Clin Oncol 2016;34:abstr 498.

22. Balar A, Bellmunt J, O'donnell PH, et al. Pembrolizumab (pembro) as first-line therapy for advanced/ unresectable or metastatic urothelial cancer: preliminary results from the phase 2 KEYNOTE-052 study. Ann Oncol 2016. doi: 10.1093/annonc/mdw435.25.

23. Ansell SM, Lesokhin AM, Borrello I, et al. PD-1 blockade with nivolumab in relapsed or refractory Hodgkin's lymphoma. N Engl J Med 2015;372:311- 9.

24. Esposito A, Criscitiello C, Curigliano G. Immune checkpoint inhibitors with radiotherapy and locoregional treatment: synergism and potential clinical implications. Curr Opin Oncol 2015;27:445-51.

25. Antonia SJ, Villegas A, Daniel D, et al. Durvalumab after Chemoradiotherapy in Stage III Non-Small-Cell Lung Cancer. N Engl J Med 2017;377:1919-29. 
26. Hess CB, Buchwald ZS, Stokes W, et al. Low-Dose Whole-Lung Radiation for COVID-19 Pneumonia: Planned Day7 Interim Analysis of a Registered Clinical Trial. medRxiv 2020. doi: 10.1101/2020.06.03.20116988

27. Jiang $X$, Wang J, Deng $X$, et al. Role of the tumor microenvironment in PD-L1/PD-1-mediated tumor immune escape. Mol Cancer 2019;18:10.

28. Bracci L, Schiavoni G, Sistigu A, et al. Immune-based mechanisms of cytotoxic chemotherapy: implications for the design of novel and rationale-based combined treatments against cancer. Cell Death Differ 2014;21:15-25.

29. Zitvogel L, Kepp O, Kroemer G. Immune parameters

Cite this article as: Shen $\mathrm{D}$, Chen $\mathrm{Q}, \mathrm{Wu} \mathrm{J}, \mathrm{Li} \mathrm{J}$, Tao K, Jiang Y. The safety and efficacy of neoadjuvant PD-1 inhibitor with chemotherapy for locally advanced esophageal squamous cell carcinoma. J Gastrointest Oncol 2021;12(1):1-10. doi: 10.21037/jgo-20-599 affecting the efficacy of chemotherapeutic regimens. Nat Rev Clin Oncol 2011;8:151-60.

30. Chen G, Emens LA. Chemoimmunotherapy: reengineering tumor immunity. Cancer Immunol Immunother 2013;62:203-16.

31. Chowdhury PS, Chamoto K, Honjo T. Combination therapy strategies for improving PD-1 blockade efficacy: a new era in cancer immunotherapy. J Intern Med 2018;283:110-20.

(English Language Editor: J. Gray) 